\title{
Achados clínico-laboratoriais de uma série de casos com endocardite infecciosa
}

\author{
Clinical and laboratory findings in a series of cases of infective endocarditis \\ Carla A.Z. Pereira ${ }^{1}$, Scheila C.G.P. Rocio ${ }^{1}$, Maria-Fátima R. Ceolin ${ }^{2}$, \\ Ana-Paula N.B. Lima ${ }^{2}$, Felippe Borlot ${ }^{3}$, Roberto S.T. Pereira ${ }^{4}$, Sandra F. Moreira-Silva ${ }^{5}$
}

\begin{abstract}
Resumo
Objetivo: Descrever os achados clínico-laboratoriais da endocardite infecciosa (EI) em 28 crianças, em Vitória/ES.

Método: Estudo retrospectivo dos prontuários de 28 crianças, com idade abaixo de 18 anos e diagnóstico de endocardite infecciosa, internadas no serviço de infectologia do Hospital Infantil Nossa Senhora da Glória, em Vitória - Espírito Santo, no período de janeiro de 1993 a dezembro de 2001. Os critérios para diagnóstico de endocardite infecciosa foram os do Duke Endocarditis Service (Duke University, Durham, North Carolina - USA): critérios maiores (hemoculturas e ecocardiograma positivos) e critérios menores (febre, doença cardíaca prévia, sopro cardíaco recente, fenômenos vasculares e imunológicos). Através de protocolo específico, preenchido pelos médicos-residentes e acadêmicos do serviço de infectologia, e revisados pelos médicos da equipe, foram anotadas as idades, sexo, achados clínicos e laboratoriais, e os resultados da ecocardiografia transtorácica das 28 crianças que preenchiam os critérios clínicos e laboratoriais. Em todos os casos, as hemoculturas foram realizadas com coleta do sangue (três amostras), sob condições assépticas e com inoculação em meios aeróbicos e anaeróbicos, incubados a uma temperatura de $37^{\circ} \mathrm{C}$ e testados com sistema automatizado Vitec System ${ }^{\circledR}$ (Biolab).
\end{abstract}

Resultados: Das 28 crianças, 16 eram do sexo masculino, com idade entre 3 e 180 meses (média 70,6 $\pm 59,2 \mathrm{~m}$ ), e a maioria (68\%) era procedente da região metropolitana de Vitória. Metade apresentava cardiopatia prévia. Os achados clínicos mais freqüentes que levaram à suspeita de EI foram: febre prolongada (100\%), sopro cardíaco $(67,9 \%)$, dispnéia $(57,1 \%)$, hepatomegalia $(57,1 \%)$, fenômenos vasculares $(32,2 \%)$, esplenomegalia $(28,6 \%)$ e nódulos de Osler $(7,1 \%)$. Foram colhidas hemoculturas dos 28 casos, sendo $16(57,1 \%)$ positivas. O S. aureus adquirido na comunidade foi o germe mais freqüentemente isolado (9/16-56,6\%). Houve persistência da febre, apesar do tratamento, duração da febre de 2,5 a 30 dias, mediana de 18,0 dias. $O$ valor médio de leucócitos à internação foi de $11.657 \pm 7.085 \mathrm{~mm}^{3}$. O ecocardiograma

\begin{abstract}
Objective: To describe clinical and laboratory data of infective endocarditis (IE) in 28 children from Vitória, state of Espírito Santo, Brazil.

Methods: We reviewed the medical records of 28 children aged 18 years and under admitted to the Infectious Diseases Unit of Nossa Senhora da Glória Children's Hospital with a diagnosis of IE from January 1993 to December 2001. The diagnosis of IE was based on the criteria established by the Duke Endocarditis Service (Duke University, Durham, North Carolina, USA): positive blood cultures and echocardiogram (primary criteria); and fever, history of heart disease, recent heart murmur, and vascular and immunological phenomena (secondary criteria). A specific protocol was filled out by interns and medical students and revised by physicians from the hospital medical team to obtain the following data: age, sex, clinical and laboratory findings, and results of transthoracic echocardiography. In all cases, three blood samples were collected under aseptic conditions and inoculated in aerobic and anaerobic environments. The samples were then incubated at $37^{\circ} \mathrm{C}$ and tested with the Vitec System ${ }^{\circledR}$ automatized system (Biolab).

Results: Among 28 patients, 16 were boys. Age was between 3 and 180 months (mean $70.6 \pm 59.2$ months). Most children (68\%) came from the metropolitan area of Vitória, and half presented a history of cardiopathy. The most frequent clinical findings which led to suspicion of IE were: prolonged fever in all cases, new or changing regurgitant murmur $(67.9 \%)$, dyspnea $(57.1 \%)$, hepatomegaly $(57.1 \%)$, vascular phenomena $(32.2 \%)$, splenomegaly $(28.6 \%)$, and Osler's nodes (7.1\%). Blood cultures were positive in 16 patients $(57.1 \%)$, and community-acquired S. aureus was the most frequently isolated pathogen (56.6\%). Despite the treatment, fever persisted for 2.5 to 30 days (median $=18.0$ days). Mean leukocyte count at admission was $11,657 \pm 7,085 \mathrm{~mm}^{3}$. Transthoracic echocardiography showed endocardial lesion in all patients, evidencing vegetations in the tricuspid valve $(25 \%)$, mitral valve $(25 \%)$, and intraventricular communication border $(28 \%)$. Thirteen $(46.4 \%)$ children
\end{abstract}

1. Médica-residente (MR3) de Infectologia Pediátrica do Hospital Infantil Nossa Senhora da Glória - Vitória/ES.

2. Médica pediatra do Serviço de Infectologia do Hospital Infantil Nossa Senhora da Glória - Vitória/ES.

3. Estudante do $4^{\circ}$ ano de Medicina da EMESCAM - Vitória/ES.

4. Médico cardiologista, chefe do Serviço de Cardiologia Pediátrica do Hospital Infantil Nossa Senhora da Glória - Vitória/ES.

5. Mestre em Doenças Infecciosas pelo Núcleo de Doenças Infecciosas/CBM-UFES e coordenadora da residência médica de Infectologia Pediátrica do Hospital Infantil Nossa Senhora da Glória - Vitória/ES. 
transtorácico, realizado em todos os pacientes, evidenciou vegetações, principalmente nas válvulas tricúspide $(25,0 \%)$, mitral $(25,0 \%)$ e na borda de CIV $(28,6 \%)$. Treze $(46,4 \%)$ crianças apresentaram sepse concomitante, e três $(10,7 \%)$ infecção hospitalar. Houve um óbito $(3,6 \%)$.

Conclusões: A endocardite infecciosa em nosso meio é freqüente em crianças abaixo de dois anos de idade e com uma cardiopatia congênita. O S. aureus de origem comunitária foi o microorganismo isolado mais freqüentemente, e em crianças sem lesão cardíaca prévia. Endocardite infecciosa aguda, endocardite bacteriana, Staphylococcus aureus, ecocardiografia transtorácica.

J Pediatr (Rio J) 2003;79(5):423-8: sobrepeso, obesidade, prevalência. presented concomitant sepsis and three (10.7\%) developed hospitalacquired infection. There was one death $(3.6 \%)$.

Conclusions: IE in our country is frequent among children younger than 2 years with congenital cardiopathy. Communityacquired $S$. aureus was the most frequently isolated microorganism in children with no previous cardiac lesion. Acute infective endocarditis, bacterial endocarditis, Staphylococcus aureus, transthoracic echocardiography.

JPediatr(RioJ) 2003;79(5):423-8: overweight, obesity, prevalence.

\section{Introdução}

A endocardite infecciosa é definida como um processo inflamatório do endocárdio valvar ou mural, sobre um defeito septal, ou sobre as cordas tendíneas, como resultado de uma infecção bacteriana, viral, fúngica, ou por micobactérias e rickéttsias, que, na maioria das vezes, ocorre em pessoas com anormalidades pré-existentes do sistema cardiovascular ${ }^{1,2}$. Compreende cerca de $0,2 \%$ a $0,5 \%$ de todas as internações pediátricas, e é considerada uma importante causa de mortalidade (20\%-64\%) entre crianças e adolescentes, apesar dos avanços do tratamento e profilaxia com agentes antimicrobianos. Vários fatores contribuem para o prognóstico da doença, como parâmetros clínicos, tipo de microorganismo e presença de prótese valvar $^{2-4}$. A doença, na ausência de lesão cardíaca prévia, é considerada rara em crianças ${ }^{5,6}$. Em adultos, é freqüente em usuários de droga intravenosa e em pessoas de 50 anos de idade ou mais ${ }^{4}$.

Em crianças, tem sido relatado aumento crescente de casos, principalmente em recém-nacidos e lactentes jovens e, concomitantemente, mudança do perfil bacteriano, com maior prevalência do Staphylococcus aureus e de outras bactérias, antes menos freqüentemente identificadas na endocardite infecciosa ${ }^{4,6}$. Nesses pacientes, a endocardite é aguda, geralmente associada a defeitos cardíacos congênitos. Os casos de endocardite subaguda clássica, causada pelo Streptococcus viridans têm diminuído, devido à redução da prevalência da febre reumática e suas seqüelas cardíacas. Além da redução de casos de febre reumática, a melhoria no atendimento de recém-nascidos de alto risco, o aumento dos procedimentos invasivos e as intervenções cirúrgicas precoces nas cardiopatias congênitas têm contribuído para o aumento do número de expostos ao risco de endocardite infecciosa nesse grupo etário ${ }^{2,4}$.

Apesar de não ser muito freqüente, a doença é grave, considerada como importante causa de morbidade e mortalidade em criança, e o sucesso terapêutico depende de diagnóstico precoce e preciso. Apesar de autores terem descrito vários critérios baseados em parâmetros clínicos e patológicos, os critérios para diagnóstico da EI mais aceitos atualmente são os critérios de Duke, que incluem os parâmetros do exame ecocardiográfico ${ }^{7,8}$ (Tabela 1). O valor desse exame tem sido questionado, mas sua validade foi confirmada quando utiliza-se ecocardiograma bidimensional com Doppler colorido, e se o cardiologista for experiente na interpretação dos resultados. Com a publicação dos novos critérios para o diagnóstico da endocardite infecciosa do serviço de endocardite infecciosa da Universidade de Duke, atualmente considera-se que os dois principais critérios para diagnóstico de certeza de endocardite infecciosa são hemoculturas múltiplas positivas para germes típicos e evidência, ao ecocardiograma, de lesões miocárdicas e/ou vegetações em válvulas cardíacas, abcessos intramiocárdios ou deiscência parcial recente de uma valva protética ${ }^{7}$.

Tabela 1 - Critérios de Duke para o diagnóstico da endocardite infecciosa ${ }^{4,7}$

\section{Critérios maiores}

- Hemocultura positiva para EI

- Microorganismo típico para EI de duas hemoculturas isoladas: Streptococcus viridans, Streptococcus bovis, grupo HACEK ou Staphylococcus aureus e Enterococcus sp adquiridos na comunidade em ausência de foco primário

- Hemoculturas persistentemente positivas

- Evidência de envolvimento endocárdico, ecocardiograma positivo para EI

- Novo sopro regurgitante

\begin{tabular}{ll}
\hline \multicolumn{1}{c}{ Critérios menores } \\
\hline$-\quad$ Predisposição: lesão cardíaca prévia \\
- \\
- Febre maior que $38^{\circ} \mathrm{C}$ \\
- & Fenômenos vasculares (ex.: lesões de Janeway) \\
$-\quad$ Evidência microbiológica: hemocultura positiva, mas sem crité- \\
rio maior
\end{tabular}

Grupo HACEK: Haemophilus sp, Actinobacillus actinomycetem comitans, Cardiobacterium hominis, Eikenella corrodens, Kingella kingae. 
O uso de ecocardiografia em adultos tem confirmado sua utilidade, especialmente com o ecocardiograma transesofágico, pois este oferece uma grande ampliação de campos para se observar imagens. Em crianças, o exame de ecocardiografia transesofágica tem uso limitado, razão pela qual utiliza-se rotineiramente o ecocardiograma transtorácico ${ }^{2,9,10}$. Como a endocardite infecciosa em crianças é uma entidade heterogênea, seu manejo requer também cuidadosos critérios baseados nas evidências clínicas e laboratoriais, além dos critérios ecocardiográficos ${ }^{11,12}$.

Como os relatos sobre o ecocardiograma transtorácico em endocardite infecciosa de crianças têm mostrado resultados variáveis na sua sensibilidade diagnóstica, nos propusemos a descrever uma série de 28 casos de endocardite infecciosa atendidas no Hospital Infantil Nossa Senhora da Glória, em Vitória, Espírito Santo, com ênfase nos achados clínicos, laboratoriais e do ecocardiograma transtorácico, para verificar as características da doença no nosso meio.

\section{Pacientes e métodos}

Foram incluídas todas as crianças com idade abaixo de 18 anos, com diagnóstico de endocardite infecciosa, internadas no serviço de infectologia do Hospital Infantil Nossa Senhora da Glória, Vitória, Espírito Santo, no período de janeiro de 1993 a dezembro de 2001. Os critérios para diagnóstico de endocardite infecciosa foram os do Duke Endocarditis Service (Duke University, Durham, North Carolina $-U S A)^{7}$, no qual um dos principais parâmetros diagnósticos é a demonstração ecocardiográfica de evidência de lesão miocárdica e/ou vegetações em válvulas cardíacas, abcessos intramiocárdicos ou deiscência parcial recente de uma valva protética.

O Hospital Infantil Nossa Senhora da Glória é um hospital público estadual, e os serviços de infectologia e de cardiologia pediátricas são referências para o Estado do Espírito Santo, atendendo também pacientes do sul da Bahia e leste de Minas Gerais.

Através de protocolo específico, preenchido pelos médicos-residentes e acadêmicos do serviço de infectologia, e revisados pelas médicas da equipe, foram anotadas as idades, sexo, achados clínicos e laboratoriais e os resultados da ecocardiografia transtorácica das 28 crianças que preenchiam os critérios clínicos e laboratoriais de endocardite infecciosa. Todos os exames laboratoriais foram realizados no laboratório de rotina do hospital. Em todos os casos, as hemoculturas foram realizadas com coleta do sangue (três amostras), sob condições assépticas e com inoculação em meios aeróbicos e anaeróbicos, incubados a uma temperatura de $37^{\circ} \mathrm{C}$ e testados com sistema automatizado Vitec System ${ }^{\circledR}$ (Biolab).

O estudo foi submetido e teve a aprovação do comitê de ética e pesquisa do Hospital Infantil Nossa Senhora da Glória.

\section{Análise estatística}

Foi feita com a utilização do programa estatístico "SPSS-CDC Inc for Windows" (Statistical Package for the Social Sciences of Centers Diseases Control - USA. Release 8.0 - 1998). A comparação das médias foi feita através do teste $t$ pareado ou pelo teste de Wilcoxon. As proporções foram comparadas pelo teste de qui-quadrado, com correção de Yates, ou pelo teste exato de Fisher.

\section{Resultados}

Os dados sobre idade, sexo, características clínicas, bacteriológicas e os principais achados do ecocardiograma estão na Tabela 2. Das cardiopatias prévias observadas, 12 eram cardiopatias congênitas $(85,7 \%)$, e duas cardiopatias reumáticas $(14,3 \%)$. Os achados clínicos que mais freqüentemente levaram à suspeita de endocardite infecciosa e induziram à realização de ecocardiograma foram: febre prolongada (100\%), sopro cardíaco $(67,9 \%)$, dispnéia $(57,1 \%)$, hepatomegalia $(57,1 \%)$, fenômenos vasculares $(32,2 \%)$, incluindo pequenas lesões eritematosas, ou hemorrágicas indolores nas palmas de mãos e plantas dos pés (lesões de Janeway), esplenomegalia $(28,6 \%)$ e pequenos nódulos intradérmicos dolorosos nas polpas dos dedos (nódulos de Osler, 7,1\%).

A hemocultura foi positiva em $16(57,1 \%)$ dos 28 casos. O Staphylococcus aureus adquirido na comunidade foi o germe mais frequientemente isolado (9/16 $56,2 \%)$. Houve diferença estatisticamente significativa na freqüência do tipo de germe isolado entre as crianças abaixo ou acima de 24 meses de idade: Staphylococcus aureus foi encontrado nos oito casos de crianças acima de 24 meses, sem lesão cardíaca prévia, e em apenas um caso no grupo com idade inferior. As outras sete crianças que tiveram hemoculturas positivas tinham idade inferior a 24 meses, e os microorganismos isolados foram: Streptococcus viridans em dois casos $(12,5 \%)$, Enterobacter $s p$ em quatro casos $(25,5 \%)$ e Candida tropicalis em um caso $(6,2 \%)$.

Houve persistência da febre, apesar do tratamento antimicrobiano, de 2,5 a 30 dias, mediana de 18,0 dias. O leucograma da internação mostrou ampla variação dos valores do número total de leucócitos (valor médio de $11.657 \pm 7.085 \mathrm{~mm}^{3}$ ), mas com evidente predomínio de neutrófilos jovens em todos os casos, se comparadas as médias dos valores dos mesmos à internação e no dia da alta hospitalar $(\mathrm{p} \leq 0,000)$ (Tabela 3$)$. O ecocardiograma transtorácico evidenciou vegetações endocárdicas em todos os casos, especialmente nas válvulas tricúspide $(25,0 \%)$ e mitral $(25,0 \%)$ e nas bordas de comunicação interventricular $(28,6 \%)$ (Figura 1). Treze $(46,4 \%)$ crianças apresentaram sepse concomitante, e três $(10,7 \%)$ desenvolveram infecção hospitalar. Ocorreu um óbito (3,6\% dos casos). 
Tabela 2 - Principais dados demográficos, clínicos, ecocardiográficos e laboratoriais observados em 28 casos de endocardite infecciosa, no HINSG, no período de 1993 a 2001

\begin{tabular}{|c|c|c|c|c|c|c|c|c|c|c|}
\hline Caso & Idade & $\begin{array}{c}\text { Sexo } \\
\text { (meses) }\end{array}$ & $\begin{array}{c}\text { Cardiopatia } \\
\text { prévia }\end{array}$ & Sepse & Sopro & Petéquias & $\begin{array}{c}\text { Hepato/ } \\
\text { Esplenomegalia }\end{array}$ & $\begin{array}{c}\text { Micro- } \\
\text { organismo }\end{array}$ & Vegetações & Evolução \\
\hline 1 & 6 & $\mathrm{~F}$ & + C. Cong. & - & + & - & + & Entero & Borda CIV & Alta \\
\hline 2 & 41 & $\mathrm{~F}$ & - & + & - & - & + & Staphilo & End. VD & Alta \\
\hline 3 & 106 & $\mathrm{~F}$ & - & + & + & - & - & Staphilo & Mitral & Alta \\
\hline 4 & 5 & $\mathrm{~F}$ & + C. Cong. & - & - & - & + & Strepto & Borda CIV & Alta \\
\hline 5 & 6 & $\mathrm{~F}$ & - & + & - & - & + & Entero & End. VE & Alta \\
\hline 6 & 5 & M & + C. Cong. & - & + & - & + & Staphilo & Borda CIV & Alta \\
\hline 7 & 132 & $\mathrm{~F}$ & - & + & - & + & + & Staphilo & Tricúspide & Alta \\
\hline 8 & 108 & M & - & + & + & + & - & Staphilo & Pulmonar & Alta \\
\hline 9 & 158 & $\mathrm{~F}$ & - & + & + & + & - & Negativo & Mitral & Alta \\
\hline 10 & 10 & M & + C. Cong. & + & + & - & + & Entero & Borda CIV & Alta \\
\hline 11 & 3 & M & - & + & - & - & - & Entero & Tricúspide & Alta \\
\hline 12 & 8 & M & + C. Cong. & + & + & - & + & C.tropic. & Borda CIV & Alta \\
\hline 13 & 156 & M & - & - & + & - & - & Negativo & Mitral & Alta \\
\hline 14 & 60 & M & + C. Cong. & - & + & - & - & Negativo & Pulmonar & Alta \\
\hline 15 & 24 & M & - & - & - & - & - & Strepto & Mitral & Alta \\
\hline 16 & 156 & M & - & + & + & - & - & Negativo & Tricúspide & Alta \\
\hline 17 & 120 & M & + C. Reum. & - & - & + & + & Negativo & Mitral & Alta \\
\hline 18 & 132 & M & - & + & + & + & + & Staphilo & Mitral & Alta \\
\hline 19 & 35 & M & - & - & - & - & + & Staphilo & Tricúspide & Alta \\
\hline 20 & 52 & M & + C. Cong. & - & + & - & + & Staphilo & Tricúspide & Alta \\
\hline 21 & 36 & $\mathrm{~F}$ & - & + & - & - & + & Staphilo & End. VD & Alta \\
\hline 22 & 24 & M & - & - & + & - & - & Negativo & Tricúspide & Alta \\
\hline 23 & 84 & $\mathrm{~F}$ & + C. Cong. & - & + & - & - & Negativo & Borda CIV & Alta \\
\hline 24 & 132 & $\mathrm{~F}$ & + C. Reum. & - & + & - & + & Negativo & Mitral & Alta \\
\hline 25 & 105 & M & + C. Cong. & - & + & - & - & Negativo & Borda CIV & Alta \\
\hline 26 & 24 & $\mathrm{~F}$ & + C. Cong. & + & + & - & + & Negativo & Tricúspide & Alta \\
\hline 27 & 3 & $\mathrm{~F}$ & + C. Cong. & - & + & - & + & Negativo & Pulmonar & Óbito \\
\hline 28 & 180 & M & + C. Cong. & - & + & - & - & Negativo & Borda CIV & Alta \\
\hline
\end{tabular}

End = endocárdio, + presente, - ausente.

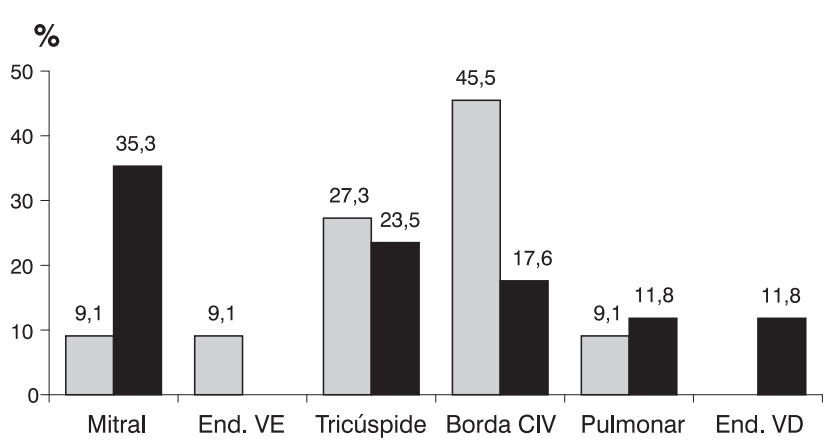

$$
\square \leq 24 \text { meses } \square>24 \text { meses }
$$

END = endocárdio, CIV = comunicação interventricular.

Figura 1 - Localização das lesões observadas no ecocardiograma, em 28 casos de endocardite infecciosa

\section{Discussão}

Tem sido relatado por vários centros cardiológicos pediátricos a observação de aumento dos casos de endocardite infecciosa, principalmente entre crianças abaixo de dois anos de idade, em decorrência do aumento da sobrevida de crianças submetidas a cirurgias de cardiopatias complexas, devido à melhor assistência prestada e às unidades de terapia intensiva ${ }^{4,6}$. Considerando que, em pediatria, a endocardite infecciosa é uma entidade heterogênea, devemos observar todos os possíveis critérios, desde achados laboratoriais, ecocardiográficos e que não excluam os já relatados como critérios clínicos definidores da doença $^{5,7,11}$, somando todos os esforços na tentativa de um diagnóstico de urgência e de certeza, para um tratamento adequado e precoce, reduzindo, assim, a mortalidade de crianças com endocardite. Publicações recentes demonstram mudanças significativas em endocardites infecciosas pediátricas, modificações de prevalência da distribuição 
Tabela 3 - Comparação dos valores da hemoglobina, leucócitos e neutrófilos em bastões em 28 casos de endocardite infecciosa, observados na internação e no dia da alta hospitalar, HINSG,1993-2001

\begin{tabular}{lcccccc}
\hline Estatística & \multicolumn{2}{c}{ Hemoglobina } & \multicolumn{2}{c}{ Leucócitos } & \multicolumn{2}{c}{ N. Bastões } \\
& Internação & Alta & Internação & Alta & Internação & Alta \\
\hline Média & 10,1 & 10,2 & 11.657 & 8.816 & 15,0 & 2,0 \\
Mediana & 10,0 & 10,0 & 10.550 & 9.000 & 9,0 & 2,0 \\
Desvio padrão & 2,2 & 2,1 & 7.085 & 2.870 & 21,0 & 2,0 \\
Amplitude: & & & & & & \\
$\quad$ Valor mínimo & 5,2 & 7,2 & 800 & 3.500 & 1,0 & 0,0 \\
$\quad$ Valor máximo & 14,8 & 16,2 & 29.000 & 15.900 & 85,0 & 7,0 \\
$\quad$ Valor de p & $0,861^{*}$ & $0,192^{*}$ & $0,000^{\dagger}$ & & & \\
\hline
\end{tabular}

* Teste $t$ pareado.

† Teste de Wilcoxon (variável sem distribuição normal).

de idades e dos microorganismos envolvidos como causadores da doença $a^{3,4,6}$.

Nossos resultados, semelhantes ao relatado na literatura, demonstraram mudança do perfil epidemiológico da endocardite infecciosa também no nosso meio, já que 50\% dos casos ocorreram em recém-nascidos e lactentes, e o Staphylococcus aureus foi frequientemente isolado nas hemoculturas positivas. Ainda que a hemocultura tenha sido positiva em apenas $57,1 \%$ dos casos, os resultados reforçam a idéia da alta prevalência de $S$. aureus como agente etiológico da endocardite infecciosa aguda no nosso meio. Por outro lado, não temos uma explicação adequada para a baixa frequiência de hemoculturas positivas nessa série de casos, tendo em vista que as coletas de sangue foram realizadas de maneira aparentemente adequada. É possível que o uso prévio de antibióticos seja o principal fator, já que muitos pacientes chegaram ao Hospital Infantil transferidos de outras unidades de saúde.

O papel do ecocardiograma transtorácico como meio diagnóstico em crianças com suspeita clínica de endocardite infecciosa ainda gera discussões, sendo assunto controverso entre vários autores $5,8,9,11$. Alguns autores o têm considerado de baixa sensibilidade como método para diagnóstico de EI em pacientes com poucos critérios clínicos da doença ${ }^{9,12}$, porém outros avaliam que o uso da ecocardiografia na avaliação de criança com suspeita clínica de endocardite tem ampla aplicação, principalmente por que, em pediatria, o eco transtorácico oferece excelente campo ${ }^{4,11}$. Neste estudo, alteração característica ao exame foi um dos critérios de inclusão. No entanto, é importante lembrar que casos com ecocardiograma transtorácico negativo e que não apresentavam todos os critérios para diagnóstico de endocardite infecciosa poderiam ter lesões de endocardite infecciosa, ainda sem evidência, e foram tratados como sepse, sendo excluídos do grupo de endocardite infecciosa.
A microbiologia das endocardites infecciosas em crianças tem mudado consideravelmente, conforme relato de alguns autores, com o decréscimo das infecções devido ao Streptococcus viridans, com surgimento de novos agentes etiológicos, incomuns em décadas passadas, e como fator importante desta mudança está a maior sobrevida de crianças com cardiopatia congênita, e também o aumento da incidência de infecções estafilocócicas ${ }^{6,11,12}$. Este fato também foi por nós observado, e os resultados demonstraram que endocardite infecciosa foi freqüente entre crianças abaixo de dois anos de idade e naquelas com cardiopatias congênitas. Demonstraram também que o Staphylococcus aureus, de origem comunitária, foi freqüentemente isolado como microorganismo infectante em crianças sem lesões cardíacas prévias.

\section{Agradecimentos}

À Dra. Alba Lília de Almeida Leite, médica pediatra do serviço de infectologia do Hospital Infantil Nossa Senhora da Glória, pelo auxílio no acompanhamento dos casos, e ao Dr. Fausto Edmundo Lima Pereira, Professor Doutor do Núcleo de Doenças Infecciosas da Universidade Federal do Espírito Santo, pelo auxílio na revisão final do texto e pelo incentivo contínuo à pesquisa e produção científica.

Aos funcionários do serviço de arquivamento de prontuários do HINSG, por sua dedicação.

\section{Referências bibliográficas}

1. Estlow MM. Prevention of infective endocarditis in the pediatric congenital heart population. J Pediatr Nurs 1998;24:205-12.

2. Karchmer AW. Endocardite infecciosa. In: Braunwald E, editor. Tratado de Medicina Cardiovascular. $5^{\mathrm{a}}$ ed. São Paulo: Roca; 1999. p. 1149-74. 
3. Mansur AJ, Grinberg M, Cardoso RHA, da Luz PL, Bellotti G, Pileggi F. Determinants of prognosis in 300 episodes of infective endocarditis. Thorac Cardiovasc Surg 1996;44:2-10.

4. Foronda A, Foronda G, Camargo LFA. Endocardite Infecciosa. In: Ebaid M, editor. Cardiologia em Pediatria. $1^{a}$ ed. São Paulo: Roca; 2000. p. 479-93.

5. Sable CA, Rome JJ, Martin GR, Patel KM, Karr SS. Indications for echocardiography in the diagnosis of infective endocarditis in children. Am J Cardiol 1995; 75:801-4.

6. Ashkenazi S, Levy O, Blieden L. Trends of childhood infective endocarditis in Israel with emphasis on children under 2 years of age. Pediatr Cardiol 1997;18:419-24.

7. Durack DT, Lukes AS, Bright DK. New criteria for diagnosis of infective endocarditis: utilization of specific echocardiographic findings. Duke Endocarditis Service. Am J Med 1994;96:200-9.

8. Bayer AS, Bolger AF, Taubert KA, Wilson W, Steckelberg J, Karchmer AW, et al. Diagnosis and management of infective endocarditis and its complications. Circulation 1998;98:2936-48.

9. Aly AM, Simpson PM, Humes RA. The role of transthoracic echocardiography in the diagnosis of infective endocarditis in children. Arch Pediatr Adolesc Med 1999;153:950-4.
10. Barreto J, Waderlinder J, Reitich J. Hallarzgos ecocardiográficos y de laboratorio en pacientes pediátricos con endocarditis infecciosa. Bol Hosp Ninos J M de los Rios 1998;34:57-60.

11. Li JS, Milazzo-Jr AS. Bacterial endocarditis in infants and children. Clin Infect Dis 2000;30:633-8.

12. Heper G, Yorukoglu Y. Clinical, bacteriologic and echocardiographic evaluation of infective endocarditis in Ankara, Turkey. Angiology 2002;53:191-7.

Endereço para correspondência:

Dra. Sandra F. Moreira-Silva

Rua Natalina Daher Carneiro, 55/302 - Jardim da Penha CEP 29060-490 - Vitória - ES

Tel. (27) 3225.6070 - Fax: (27) 3137.2415 / 3137.2477

E-mail: sanfag@terra.com.br 